\title{
All that Glitters Is not Gold: Consumer Health Informatics and Education in the Era of Social Media and Health Apps
}

\section{Findings from the Yearbook 2016 Section on Consumer Health Informatics}

\author{
L. Fernandez-Luque ${ }^{1}$, P. Staccini ${ }^{2}$, Section Editors for the IMIA Yearbook Section on \\ Consumer Health Informatics \\ 1 Qatar Computing Research Institute, HBKU, Qatar Foundation, Doha, Qatar \\ 2 INSERM UMR 912 SESSTIM, IRIS Dept, UFR Médecine, Université Nice-Sophia Antipolis, France
}

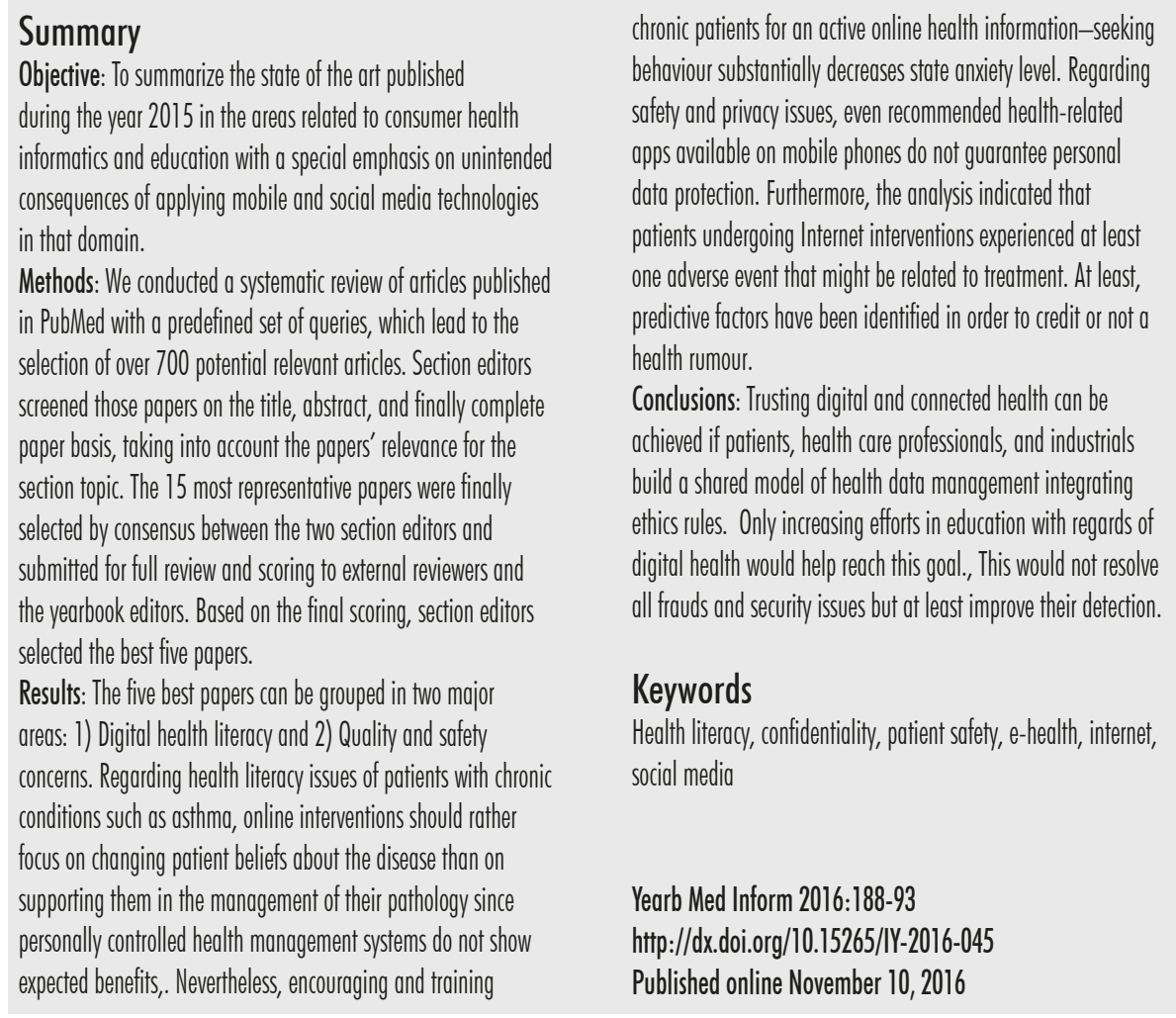

\section{Introduction}

Internet and mobile technology are widely used in the health domain and numerous studies have reported that digital health intervention, if properly designed, can increase the quality of life of patients and also improve the quality of health care. Furthermore, new technologies are increasingly being used to enhance the education of health care professionals and patients. Nevertheless, despite all those potential benefits, many challenges are arising. The unintended consequences of the use of health information technology (IT) are very complex and span from wasting resources in failed digital health interventions, to harming patients (includ- ing privacy leaks) and increasing the gap in health disparities.

Unintended consequences of health IT have been a major topic in medical informatics conferences during 2015, especially with regards to patient safety, which is becoming a major area of research. For example, in Medinfo 2015, Virginio L. described the patient risk associated with Electronic Health Records [1]. Very active Working Groups of the medical informatics community are studying this problem, such as the Patient Safety Group of IMIA (Health Informatics for Patient Safety), and consumer health and education groups. Moreover, media attention is also increasing in this topic. For example, in China an investigation has been conducted on the web-search giant Baidu to identify its role in the promotion of misinformation which apparently would have contributed to the death of a cancer patient [2].

As in previous years, section editors have reviewed the literature in the area of consumer health informatics and education. In 2016, the IMIA Yearbook editorial board selected as special topic the Unintended Consequences of Health IT. As explained before, this is a topic of growing interest in the area covered by this section. This synopsis reports on the best papers published in 2015 that provide a wide spectrum of unintended consequences of health IT use for consumer health informatics and education. 


\section{Methodology}

Section editors used PubMed to conduct the review. Following the methodology from previous years [3], they used the following query to capture relevant papers about consumer health informatics and education. This query retrieved 721 results.

"(((2015[DP] NOT pubstatusaheadofprint)) NOT Bibliography[pt] NOT Comment[pt] NOT Editorial[pt] NOT Letter[pt] NOT News[pt] NOT Case Reports[pt] NOT Published Erratum[pt] NOT Historical Article[pt] NOT Legal Cases[pt] NOT legislation[pt] NOT ("review"[Publication Type] OR "review literature as topic"[MeSH Terms] OR "literature review"[All Fields])) “

AND ("people with"[All Fields] OR "patient centered care"[All Fields] OR "care coordination"[All Fields] OR "consumer health informatics"[All Fields] OR "health information"[All Fields] OR "consumer health information"[All Fields] OR "consumer product safety"[All Fields] OR "consumer satisfaction"[All Fields] OR "patient safety"[All Fields] OR "patient satisfaction"[All Fields] OR "personal health"[All Fields] OR "personalized medicine"[All Fields] OR "patient-reported outcomes"[All Fields])

AND ("ehealth"[All Fields] OR "e-health"[All Fields] OR "telehealth"[All Fields] OR "mhealth"[All Fields] OR "telemedicine"[All Fields] OR "electronic patient-physician communication" All Fields] OR "personal health record"[All Fields] OR "electronic patient record"[All Fields] OR "online"[All Fields] OR "electronic"[All Fields] OR "information technology"[All Fields] OR "communication technology"[All Fields] OR "mobile"[All Fields] OR "on-line"[All Fields] OR "online systems"[All Fields] OR "patient portal"[All Fields] OR "decision support technique"[All Fields] OR "health care access"[All Fields] OR "online health information seeking"[All Fields] OR "health information systems"[All Fields])

AND ("internet"[All Fields] OR "web"[All Fields] OR "website"[All Fields] OR "social media"[All Fields] OR "facebook"[All Fields] OR "twitter"[All Fields] OR "youtube"[All Fields] OR "social network site"[All Fields] OR "social web"[All

Table 1 Best paper selection of articles for the IMIA Yearbook of Medical Informatics 2016 in the section 'Consumer Health Informatics'. The articles are listed in alphabetical order of the first author's surname.

\section{Section}

Consumer Health Informatics

- Huckvale K, Prieto JT, Tilney M, Benghozi P-J, Car J. Unaddressed privacy risks in accredited health and wellness apps: a cross-sectional systematic assessment. BMC Med 2015;13(1):214.

- Lau AY. Why Didn't it Work? Lessons From a Randomized Controlled Trial of a Web-based Personally Controlled Health Management System for Adults with Asthma. J Med Internet Res 2015;17: e283.

- Minto C, Bauce B, Calore C, Rigato I, Folino F, Soriani N, Hochdorn A, lliceto S, Gregori D. Is Internet use associated with anxiety in patients with and at risk for cardiomyopathy? Am Heart J 2015;170(1):87-95.

- Rozental A, Boeftcher J, Andersson G, Schmidt B, Carlbring P. Negative Effects of Internet Interventions: A Qualitative Content Analysis of Patients' Experiences with Treatments Delivered Online. Cogn Behav Ther 2015;44(3):223-36.

- Zhang Z, Zhang Z, Li H. Predictors of the authenticity of Internet health rumours. Health Info Libr J 2015;32(3):195-205.

Fields] OR "online social network"[All Fields] OR "social environment"[All Fields] OR "social process" [All Fields] OR "social competition"[All Fields] OR "social norm"[All Fields] OR "social feedback"[All Fields] OR "social influence"[All Fields] OR "social comparison"[All Fields] OR "social network"[All Fields] OR "discussion group"[All Fields] OR "support group"[All Fields] OR "social support"[All Fields] OR "community network"[All Fields])

The first query was complemented by another query in order to capture the publications related to the unintended consequences of the use of information and communications technology (ICT) on consumer health and education. This query retrieved 179 results. The full query is reproduced below.

(2015[DP] NOT (pubstatusaheadofprint) NOT("Bibliography"[Publication Type] OR "Comment"[Publication Type] OR "Editorial"[Publication Type] OR "Letter"[Publication Type] OR "News"[Publication Type] OR "Case Reports"[Publication Type] OR "Published Erratum"[Publication Type] OR "Historical Article"[Publication Type] OR "Legal Cases"[Publication Type] OR "legislation"[Publication Type] OR "review"[Publication Type] OR "review literature as topic" [MeSH Terms] OR "literature review"[All Fields] OR "systematic review"[All Fields])) AND ("consumer health informatics"[All Fields] OR "consumer health information"[All Fields] OR "consumer"[All Fields] OR "health information"[All Fields] OR "health informatics"[All Fields])
AND ("consequence"[All Fields] OR "impact"[All Fields] OR "disparities"[All Fields] OR "inequality"[All Fields] OR "effect"[All Fields] OR "issue"[All Fields] OR "risk"[All Fields] OR "emotion"[All Fields] OR "ethics"[All Fields] OR "unintended"[All Fields] OR "unexpected"[All Fields] OR "unsafety"[All Fields] OR "unsatisfaction"[All Fields] OR "misusage"[All Fields] OR "misinformation"[All Fields] OR "unacceptance"[All Fields] OR "harm"[All Fields] OR "privacy"[All Fields]) AND ("ehealth"[A11 Fields] OR "e-health"[All Fields] OR "telehealth"[All Fields] OR "mhealth"[All Fields] OR "telemedicine"[All Fields] OR "electronic patient-physician communication"[All Fields] OR "personal health record"[All Fields] OR "electronic patient record"[All Fields] OR "online"[All Fields] OR "electronic"[All Fields] OR "information technology"[All Fields] OR "communication technology"[All Fields] OR "mobile"[All Fields] OR "on-line"[All Fields] OR "online systems"[All Fields] OR "patient portal"[All Fields] OR "decision support technique"[All Fields] OR "health care access"[All Fields] OR "online health information seeking"[All Fields] OR "health information systems"[All Fields] OR "internet intervention"[All Fields])

AND ("internet"[All Fields] OR "web"[All Fields] OR "website"[All Fields] OR "social media"[All Fields] OR "facebook"[All Fields] OR "twitter"[All Fields] OR "youtube"[All Fields] OR "social net- 
work site"[All Fields] OR "social web"[All Fields] OR "online social network"[All Fields] OR "social environment"[All Fields] OR "social process"[All Fields] OR "social competition"[All Fields] OR "social norm"[All Fields] OR "social feedback"[All Fields] OR "social influence"[All Fields] OR "social comparison"[All Fields] OR "social network"[All Fields] OR "discussion group"[All Fields] OR "support group"[All Fields] OR "social support"[All Fields] OR "community network"[All Fields] OR "peer group"[All Fields])

Section editors reviewed all returned papers and selected a list of 15 potential best papers. Five selection criteria have been used: level of relevance regarding the 2016 yearbook special topic "Unintended consequences of Health IT"; level of evidence of the results; design of the study: cohort analysis or intervention assessment; nature of the problem addressed: impact of public health intervention or patient's literacy. Then the 15 papers were blind reviewed by an international group of external experts according to the Yearbook selection process. During a consensus meeting, section editors finally selected the five best papers to be included in the section.

\section{Results}

The selected 15 papers and the top five best papers can be grouped in two major areas: 1) Digital Health Literacy and 2) Quality and Safety Concerns.

\section{Digital Health Literacy}

One of the major aspects discussed in several of the 15 papers included the impact of uneven digitalization with regards of health disparities, specially with regards of elderly citizens [4-7]. These studies highlight the importance of digital health literacy for both professionals and patients in order to minimise the risk of using Internet. Other papers analysed specific cases of health consumers using internet as part of their decision-making and support seeking [8-12]. In all these studies, the importance of education was highlighted. In fact, in another study, Annie Lau et al. reported the problems faced in a clinical trial assessing an online intervention for self-management of asthma [13]. Consistently with the studies about digital health literacy, the problems faced in that study were related to the skills needed by patients to use the system.

The need for enhancing digital health literacy is not specific to health consumers. It also applies to healthcare professionals [14]. For example, A. Barry et al. reported about the increasing use of social media by pharmacists and the challenges they have to face in doing so.

The selected best papers in this group are: - Lau AY. Why Didn't it Work? Lessons From a Randomized Controlled Trial of a Web-based Personally Controlled Health Management System for Adults with Asthma [Internet]. J Med Internet Res 2015;27:e283.

- Minto C, Bauce B, Calore C, Rigato I, Folino F, Soriani N, Hochdorn A, Iliceto $\mathrm{S}$, Gregori D. Is Internet use associated with anxiety in patients with and at risk for cardiomyopathy? Am Heart J 2015;170(1):87-95.

\section{Quality and Safety Concerns}

The quality of online health information was, again, a cause of concern during 2015. Several of the 15 preselected papers analysed the issues related to that. For example, researchers in China proposed methods allowing to identify health rumours [15]. Another study studied the spreading of rumours against the use of fluoride [16]. In 2015, another paper reported the results of a systematic review about the negative effects of online health interventions [17]. They found that nearly $10 \%$ of the participants to studies reported negative consequences.

The quality of health apps is also becoming crucial since the penetration of smartphones is significantly growing and health care professionals and consumers are widely using them. In a shocking study, Kuckvale et al. found serious security threats in health and fitness apps recom- mended to the public by a governmental white list (UK NHS Health Apps Library) [18]. Most of the apps did not follow basic encryption standards thus posting a major threat to the privacy of patients that were using them.

The selected best papers in this group are:

- Huckvale K, Prieto JT, Tilney M, Benghozi P-J, Car J. Unaddressed privacy risks in accredited health and wellness apps: a cross-sectional systematic assessment. BMC Med 2015;13(1):214

- Rozental A, Boettcher J, Andersson G, Schmidt B, Carlbring P. Negative Effects of Internet Interventions: A Qualitative Content Analysis of Patients' Experiences with Treatments Delivered Online. Cogn Behav Ther 2015;44(3):223-36.

- Zhang Z, Zhang Z, Li H. Predictors of the authenticity of Internet health rumours. Health Info Libr J 2015;32(3):195-205.

\section{Conclusions}

In order to minimize the risk of using Health IT a special effort needs to be done in improving the digital health literacy of patients and health care professionals. This is especially true in the case of populations already suffering from health disparities since new technologies may increase these health disparities.

The quality of online and health apps is also a serious concern. One approach to minimize the impact of misleading health information is the training of patients and health care professionals to improve their skillset so that they are able to make the difference between high quality content and low quality content on the net. In addition, new research is being conducted to automatically identify misleading information (e.g. rumours) in order to help patients and health care professionals in their decision-making process.

However, education cannot solve everything. Neither patients nor professionals can easily identify if a mobile app is encrypting their health information or not.

In addition to the traditional concerns with regards to the quality of the informa- 
tion, new threats are appearing such as the lack of security when transmitting health information in mobile apps. Findings with regards to the lack of basic security standards in the health apps listed in the UK NHS Health Apps Library is going to raise a major debate in multiple areas such as trust, legal responsibility, enforcement of recommendations, etc.

\section{References}

1. Virginio LA, Ricarte ILM. Identification of Patient Safety Risks Associated with Electronic Health Records: A Software Quality Perspective. Stud Health Technol Inform 2015;216:55-9.

2. Ramzy A. China Investigates Baidu After Student's Death From Cancer. The New York Times 2016 May.

3. Staccini P, Fernandez-Luque L. Health Social Media and Patient-Centered Care: Buzz or Evidence? Findings from the Section "Education and Consumer Health Informatics" of the 2015 Edition of the IMIA Yearbook. Yearb Med Inform 2015:160-3.

4. Bailey SC, O'Conor R, Bojarski EA, Mullen R, Patzer RE, Vicencio D, Jacobson KL, Parker RM, Wolf MS.. Literacy disparities in patient access and health-related use of Internet and mobile technologies. Heal Expect 2015;18(6):3079-87.

5. Feng Y, Xie W. Digital divide 2.0: the role of social networking sites in seeking health information online from a longitudinal perspective. J Health Commun 2015;20(1):60-8.

6. Levy H, Janke AT, Langa KM. Health literacy and the digital divide among older Americans. J Gen Intern Med 2015;30(3):284-9.

7. Norman C. eHealth literacy 2.0: problems and opportunities with an evolving concept. J Med Internet Res 2011;13(4).

8. Magnezi R, Grosberg D, Novikov I, Ziv A, Shani M, Freedman LS. Characteristics of patients seeking health information online via social health networks versus general Internet sites: a comparative study. Inform Health Soc Care 2015;40(2):125-38.

9. Jamal A, Khan SA, AlHumud A, Al-Duhyyim A, Alrashed M, Bin Shabr F, Alteraif A, Almuziri A, Househ M, Qureshi R.. Association of Online Health Information-Seeking Behavior and SelfCare Activities Among Type 2 Diabetic Patients in Saudi Arabia. J Med Internet Res 2015;17(8):e196.

10. Minto C, Bauce B, Calore C, Rigato I, Folino F, Soriani N, Hochdorn A, Iliceto S, Gregori D.. Is Internet use associated with anxiety in patients with and at risk for cardiomyopathy? Am Heart J 2015;170(1):87-95, 95.e1-4

11. Mohd Roffeei SH, Abdullah N, Basar SKR. Seeking social support on Facebook for children with Autism Spectrum Disorders (ASDs). Int J Med Inform 2015;84(5):375-85.

12. Paolino L, Genser L, Fritsch S, De' Angelis N, Azoulay D, Lazzati A. The web-surfing bariatic pa- tient: the role of the internet in the decision-making process. Obes Surg 2015;25(4):738-43.

13. Lau AY. "Why Didn't it Work?" Lessons From a Randomized Controlled Trial of a Web-based Personally Controlled Health Management System for Adults with Asthma. J Med Internet Res 2015;17:e283.

14. Barry AR, Pearson GJ. Professional use of social media by pharmacists. Can J Hosp Pharm 2015 [cited 2016 May 26];68(1):22-7.

15. Zhang Z, Zhang Z, Li H. Predictors of the authenticity of Internet health rumours. Health Info Libr J England 2015 Sep;32(3):195-205.

16. Seymour B, Getman R, Saraf A, Zhang LH, Kalenderian E. When advocacy obscures accuracy online: Digital pandemics of public health misinformation through an antifluoride case study. Am J Public Health 2015;105(3):517-23.

17. Rozental A, Boettcher J, Andersson G, Schmidt B, Carlbring P. Negative Effects of Internet Interventions: A Qualitative Content Analysis of Patients' Experiences with Treatments Delivered Online. Cogn Behav Ther 2015;44(3):223-36.

18. Huckvale K, Prieto JT, Tilney M, Benghozi P-J, Car $\mathrm{J}$. Unaddressed privacy risks in accredited health and wellness apps: a cross-sectional systematic assessment. BMC Med 2015;13(1):214.

\section{Correspondence to:}

Luis Fernandez-Luque, PhD

Qatar Computing Research Institute, Hamad Bin Khalifa

University

Qatar Foundation,

HBKU Research Complex, Doha, Qatar

E-mail: lluque@qf.org.qa

\section{Appendix: Content Summa- ries of Selected Best Papers for the IMIA Yearbook 2016}

\section{Lav AY \\ Why Didn't it Work? Lessons From a Randomized Controlled Trial of a Web-based Personally Controlled Health Management System for Adults with Asthma}

\section{J Med Internet Res 2015; 17: e283}

Despite personally controlled health management systems (PCHMS) have been advocated as the next-generation technology to improve health behaviours and outcomes, especially for chronic illnesses, the risk they will not be adopted remains substantial. In this randomized controlled trial, authors examined how effective a PCHMS is in encouraging adults with asthma (a chronic condition) to obtain a written asthma action plan (AAP) from a health care professional and if this would lead to an improvement in asthma control. Recruitment was conducted over a period of 5 months between April and August 2013, during which 485 participants were assessed for eligibility. Recruitment was complete in August 2013 and follow-up conducted between April and June 2014. Eligible participants were adults (aged 18 years and older) living in Australia diagnosed with asthma, who had at least monthly access to the Internet and email, and had sufficient English language skills. In all, 330 participants were assessed eligible and randomized (intervention: $n=154$; control: $n=176$ ). The intervention was based on the Healthy.me Web-based PCHMS which provided participants with evidence-based information about asthma, the importance of a written AAP, and ways of obtaining a plan from a health care professional. Additionally, participants received monthly email reminders about the various interactive features of Healthy.me (eg, forum, poll, personal health record). Participants who had been randomly allocated to the control arm were redirected to a static webpage with links to patient websites (eg, the Asthma Foundation, HealthInsite, myDR) that provided educational information on asthma. The primary outcome was "possession or not of a written AAP". Secondary outcomes were related to the use of the AAP and clinical findings such as asthma exacerbation, asthma control, worsening of asthma, and loss days from work or study. As a result, access to the Healthy.me PCHMS did not improve the rate of possession of written AAPs, planned visits to health services for non-emergency asthma management, asthma status, control, or work and study productivity. Some participants suggested that they saw little benefit in using the system, either because they or their health professionals saw little value in having an AAP, because asthma management was not a major priority in their life compared to other competing priorities, or that they have already developed their own strategies to manage the condition and needed no further assistance. Perhaps as suggested by some participants, the intervention would be more helpful for those who are newly diagnosed with asthma. 
As a conclusion, the authors provided four lessons to design consumer ehealth services: 1) Consumers must perceive the need for assistance with a task; 2) Consumers must assign priority to the tasks supported by the intervention; 3) The cost of adoption of the intervention must be lower than the benefit; 4) Outcome measures must be relevant to consumers and providers. Authors admitted they were not fully understanding why some online interventions work and others do not. In this study, they identified that participant goals were poorly aligned with the clinical goals of the system despite there was clear evidence they were underpinning these latter clinical goals. They suggested that a different approach was required in the domain of asthma management in adults, at least as far as AAPs were concerned, focusing not so much on task support than on belief change.

\section{Minto C, Bauce B, Calore C, Rigato I, Folino F, Soriani N, Hochdorn A, lliceto S, Gregori D \\ Is Internet use associated with anxiety in patients with and at risk for cardiomyopathy?}

\section{Am Heart J 2015;170(1):87-95}

The aim of this study was to determine the relation between online health information seeking and anxiety level among a sample of patients with manifested cardiomyopathy or at risk for cardiomyopathy, trying to understand if such type of information and behaviour is associated with higher or lower anxiety-related psychological symptoms, in other terms trying to show whether this type of behaviour is able to improve or worsen patient's psychological condition. The research was a cross-sectional study conducted in the University Hospital of Padova, at the specialist ambulatories for cardiomyopathies, in the period between May and July 2014. Padova Cardiology Department is a national referral centre for the treatment of cardiomyopathies, enrolling patients from many parts of Italy. Before medical examination, patients completed three different questionnaires: Use of Internet Health Information (UIH) questionnaire about the use of Internet, Short form SF-12 items questionnaire on quality of life, and State-Trait Anxiety Inventory (STAI) measuring general anxiety levels. Additional information was collected about gender, age, educational level, job, type of cardiomyopathy or predisposition, pharmacological therapy, and Implantable Cardioverter Defibrillator (ICD) treatments. Study population included 104 subjects, among which 48 had a diagnosis of primary cardiomyopathy for genetic, mixed, and acquired causes or a diagnosis of secondary cardiomyopathy. The main result was that a significant association, confirmed also after adjustment, is shown between UIH score and state anxiety level: for both groups of patients with cardiomyopathy and at risk for cardiomyopathy, online health information-seeking behaviour substantially decreased state anxiety level. Patients with a higher Internet usage reported a three-point difference of anxiety levels ( 37 vs $34, \mathrm{P}=$ .041) lower than those with a less intense Internet usage. Decrease was nonlinear and happened only for UIH scores higher than approximately 50 (95\% CI 44-53). Authors noticed that this result seems to be consistent with other studies on Internet social support in relation to psychological distress and coping abilities among cancer patients.

\section{Huckvale K, Prieto JT, Tilney M, Benghozi P-J, Car J \\ Unaddressed privacy risks in accredited health and wellness apps: a cross-sectional systematic assessment}

\section{BMC Med 2015;13(1):214}

The purpose of this study was to assess to which extend accredited apps adhered to data protection principles concerning information privacy. All apps published on the NHS Health Apps Library in July 2013, targeting Android and IOS, were reviewed. Apps were excluded if they could not be downloaded or if they were more expensive than 50 USD. Final included apps were tested during a 6-month period of evaluation from August 2013 to January 2014. They were assessed for compliance with recommended practice for information collection, transmission, and mobile-device storage; confidentiality arrangements in apps and developer-provided online services; the availability and content of privacy policies; and the agreement between policies and observed behaviour. 79 apps were selected for assessment. Over half of them included functions in which personal details, health-related information, or both, were transferred to online services, and a fifth of such apps, and two-thirds of apps overall, did not have a privacy policy. In this respect, health apps, whether accredited or not, appear to be little better than non-medical apps available through general app stores, despite greater potential sensitivity surrounding health-related information. While most, but not all, privacy policies explained how information would be used, coverage of other aspects that would enable a user to make an informed choice about which information to disclose was less consistent. Half of the apps were transmitting user account details and sending unencrypted usernames and passwords. No apps encrypted local data stores, despite the widespread use of PIN or password security within apps that might reasonably lead a user to believe his/her information was protected. The observed variation prompted questions about the coverage and consistency achieved by the certification process. The findings highlighted potential shortcomings of an accreditation approach that, in respect of privacy at least, appeared to rely mainly on self-declared compliance. Regulators should consider establishing standards for accreditation processes, and be ready to intervene if accreditation programs cannot manage risks effectively. If patients or the public are deterred from using apps because of trust issues, then the potential clinical benefits of mobile health will not be realized.

\section{Rozental A, Boeftcher J, Andersson G, Schmidt B, Carlbring $P$}

\section{Negative Effects of Internet Interventions: A Qualitative Content Analysis of Patients' Experiences with Treatments Delivered Online}

\section{Cogn Behav Ther 2015;44(3):223-36}

Although numerous clinical trials have revealed promising results for the use of Internet interventions, they may not be suitable for all patients. The emergence of new symptoms, the deterioration of health condition, and the non-response to treatment were especially evident, but other types of negative effects were also described, most 
notably social stigmatization and difficulties to understand a treatment rationale. The objective of this study was to assess if and how patients undergoing Internet interventions experience negative effects in order to understand their own understanding of these events, how negative effects might affect patient well being, and whether they might have any long-term consequences. Patients were recruited from four clinical trials investigating the efficacy or effectiveness of Internet interventions for a variety of psychiatric disorders and procrastination: 130 patients with social anxiety disorder or panic disorder received guided or unguided internet cognitive behaviour therapy (ICBT) supplemented by a smartphone application; 143 patients with major depressive disorder received guided ICBT or physical activity; 135 patients with social anxiety disorder received guided ICBT with or without the addition of cognitive bias modification; 150 patients with chronic and severe procrastination received guided or unguided ICBT. Of the 558 patients who were included in the study, $52(9.3 \%)$ indicated that they had experienced at least one adverse event that might be related to treatment. Both patient- and treatment-related negative effects emerged, involving adverse events that were common during treatment and were perceived as personally distressing for patients - most notably, deterioration and the emergence of novel symptoms - similar to the adverse events found in investigations of face-to-face treatments. Many of the negative effects associated with becoming more aware of one's difficulties and experiencing different symptoms were temporary, and in a number of instances, they were perceived as meaningful in the long run. Negative effects related to the treatment also existed, consisting primarily of difficulties with the implementation of assignments and with aspects of the treatment format perceived as negative by many of the patients, particularly the pressure to complete the treatment on time. Authors suggested that flexibility in relation with the treatment program, enhanced communication, and prolonging the treatment period could be important in order to prevent some of the negative effects that were reported by patients in the current study. They recommend monitoring negative trends on standard outcome measures, as well as self-report measures probing for adverse events, in order to prevent and reverse deterioration and dropout among patients undergoing treatments via the Internet.

\section{Zhang Z, Zhang Z, Li H \\ Predictors of the authenticity of Internet health rumours}

\section{Health Info Libr J 2015;32(3): 195-205}

As the Internet can bring individuals into direct communication, disseminating rumours is easier than with traditional media, especially in public health, and false rumours are becoming new concerns. The aim of this study was to explore whether online health rumours contain any red flags that can alert about falsehood. More specifically, this study aimed at capturing the associations between the authenticity of health rumours and some indicators of the rumours themselves, including the lengths of rumour headlines and statements, the presence of certain features in rumour statements and the type of the rumour - wish or dread.
This study collected and analysed rumours listed on www.liuyanbaike.com, which is a preeminent online resource for verifying and debunking rumours in China. On 711 health-related rumour records reported on Liuyanbaike.com during February 2014, only 453 were identified as health rumours to be analysed. Of the total rumours, $9.7 \%$ contained people's names or place names, and $32.5 \%$ contained numbers, indicating that such information was common for health rumours. Furthermore, $32.5 \%$ of rumours provided information source cues, and $14.3 \%$ referenced a foreign information source. Of these rumours, $3.1 \%$ contained pictures and $5.1 \%$ contained hyperlinks, suggesting that a small proportion of rumourmongers have taken advantage of the Internet. Finally, wish rumours accounted for $40.8 \%$ of all rumours. The regression results showed that the number of characters in a rumour's headline and statement was significantly related to rumour veracity with a decrease of the probability of a rumour to be true as the length of rumour headlines and statements increased. Having a number, a source cue, or a hyperlink in rumour statement was significantly and positively related to the probability of a rumour being true. Wish rumours were more likely to be false than dread rumours. Having a picture in a rumour statement was negatively related to the probability that the rumour was true. For individuals, this study could be readily used to promote false rumour awareness and to improve the "self-defence" skills of online users against misleading health information. For health authorities, this study could assist them in clarifying rumours. 